\title{
Concerns on the risk of Malaysian civil and defense systems due to intentional electromagnetic interference
}

\begin{abstract}
Since the beginning of twenty-first century, Malaysian civil and defense systems became increasingly dependent on sophisticated electronic and wireless systems. This led to the use of many intelligent applications and emerging the smart cities which highly rely on modern technologies such as smart telecommunication, smart grid, smart transportation, artificial intelligence, smart health, enterprise Internet of Things, smart building management systems and smart governance. These introduced new services and practices which highly impacting the planning, policy, development, and operation of infrastructure of society, while co-exist with urban facilities, and must be electromagnetic compatible with the existing systems. Hence, it initiated Intentional Electromagnetic Interference which could be a significant threat towards civil, defense, industrial, and commercial sectors, with possible targets of radio and television, telecommunication networks, grid power networks, railway networks, air traffic control, government and banking administrative networks, or even defense system as well. The susceptibility and vulnerability of these sensitive systems due to the electromagnetic interference in the current decades will keep of increasing due to the expansion and spread of radiation sources.. Threats from high power microwave sources and high power electromagnetic pulses and high-altitude electromagnetic pulse on civil and defense infrastructure that include an electronic equipment and wireless systems could be harmful and may cause disturbances and damages on electronic devices and wireless systems. This paper presents an overview of the important events in history related to the high power electromagnetic sources that is paving the way for enhancing the understanding of electromagnetic interference capabilities in disturbing modern infrastructure systems. Several major incidents reported in the literature, which are suspected to have been caused by electromagnetic interference, were also analyzed.
\end{abstract}

Keyword: Electromagnetic (EM); Electromagnetic interference (EMI); Electromagnetic compatibility (EMC); High-altitude electromagnetic pulse (HEMP); High power electromagnetic (HPEM); High power microwave (HPM); Intentional electromagnetic interference (IEMI) 